\title{
ProminTools: Shedding light on proteins of unknown function in biomineralization with user friendly tools illustrated using mollusc shell matrix protein sequences
}

\author{
Alastair W Skeffington ${ }^{\text {Corresp., } 1}$, Andreas Donath ${ }^{1}$ \\ ${ }^{1}$ Max Planck Institute of Molecular Plant Physiology, Potsdam, Germany \\ Corresponding Author: Alastair W Skeffington \\ Email address: skeffington@mpimp-golm.mpg.de
}

Biominerals are crucial to the fitness of many organism and studies of the mechanisms of biomineralization are driving research into novel materials. Biomineralization is generally controlled by a matrix of organic molecules including proteins, so proteomic studies of biominerals are important for understanding biomineralization mechanisms. Many such studies identify large numbers of proteins of unknown function, which are often of low sequence complexity and biased in their amino acid composition. A lack of user-friendly tools to find patterns in such sequences and robustly analyse their statistical properties relative to the background proteome means that they are often neglected in follow-up studies. Here we present ProminTools, a user-friendly package for comparison of two sets of protein sequences in terms of their global properties and motif content. Outputs include data tables, graphical summaries in an html file and an R-script as a starting point for data-set specific visualizations. We demonstrate the utility of ProminTools using a previously published shell matrix proteome of the giant limpet Lottia gigantea. 
1 ProminTools: Shedding light on proteins of unknown function in biomineralization

2 with user friendly tools illustrated using mollusc shell matrix protein sequences

3

4 Alastair William Skeffington ${ }^{1}$, Andreas Donath ${ }^{1}$

$5 \quad{ }^{1}$ Max Planck Institute of Molecular Plant Physiology, Am Mühlenberg 1, Potsdam-Golm,

6 14476, Germany

7 Corresponding author:

8 Alastair Skeffington $^{1}$

9 Max Planck Institute of Molecular Plant Physiology, Am Mühlenberg 1, Potsdam-Golm, 14476,

10 Germany

11 Email address: skeffington@mpimp-golm.mpg.de 


\section{Abstract}

13 Biominerals are crucial to the fitness of many organism and studies of the mechanisms of

14 biomineralization are driving research into novel materials. Biomineralization is generally

15 controlled by a matrix of organic molecules including proteins, so proteomic studies of

16 biominerals are important for understanding biomineralization mechanisms. Many such studies

17 identify large numbers of proteins of unknown function, which are often of low sequence

18 complexity and biased in their amino acid composition. A lack of user-friendly tools to find

19 patterns in such sequences and robustly analyse their statistical properties relative to the

20 background proteome means that they are often neglected in follow-up studies. Here we present

21 ProminTools, a user-friendly package for comparison of two sets of protein sequences in terms

22 of their global properties and motif content. Outputs include data tables, graphical summaries in

23 an html file and an R-script as a starting point for data-set specific visualizations. We

24 demonstrate the utility of ProminTools using a previously published shell matrix proteome of the

25 giant limpet Lottia gigantea. 
28

29

30

31

32

\section{Introduction}

Mineralized structures are formed by many organisms across the tree of life including bacteria, metazoans, plants and algae (Skinner \& Jahren 2007). These biominerals are critical for fitness, playing roles in support, defence, buoyancy, regulation of ion budgets and orientation among others. Proteins have been found to be associated with many biominerals, and are hypothesised to have a key role in mineral synthesis (Evans 2019a; Evans 2019b; Wang \& Nilsen-Hamilton 2012). In some cases the roles of such proteins is relatively well understood, and some of the best studies examples come from molluscs (Song et al. 2019). For example, the proteolytic products of the Pif protein in molluscs have been shown to bind $\mathrm{CaCO}_{3}$ crystals and induce formation of the aragonite polymorph of $\mathrm{CaCO} 3$ in vitro (Suzuki et al. 2009). Knock-down of the Pif gene results in disordered growth of the aragonite crystals in the nacreous layer of the shell. In other systems, well studies examples include Amelogenin from tooth enamel, Silicatein from sponge spicules and Mms6 from magnetosome synthesising bacteria (Wang \& NilsenHamilton 2012). However in the majority of cases the function of biomineral associated proteins remains elusive.

A common workflow in biomineralization research is to first clean a mineral preparation using detergents or oxidizing agents to remove loosely associated organic matter, and subsequently to dissolve the mineral, releasing tightly mineral-associate proteins into solution that can then be analysed using proteomic methods (Marie et al. 2013b). It is generally hypothesised that these proteins are likely to be involved in mineralization, and that proximity to the site of mineralization results in their incorporation into the mineral as it grows. Some of the proteins identified may have homology to proteins of known function or recognisable domains strongly suggestive of a certain function. For example, carbonic anhydrases have been found associated 
51 with calcium carbonate minerals in several organisms (Le Roy et al. 2014) and may aid

52 generation of bicarbonate as a substrate for calcification. However there are generally many

53 proteins in such data sets which lack similarity to proteins of known function (e.g. Jackson et al.

54 2015; Kotzsch et al. 2016; Mann et al. 2006). Intriguingly, these proteins of unknown function

55 often display unusual primary sequence characteristics, such as low complexity, biased

56 composition and a high degree of predicted intrinsic disorder.

57 Informatic tools which allow biologists to easily investigate the global features of groups of

58 proteins of unknown function relative to the background proteome are currently lacking. Thus

59 many studies restrict their analysis of these proteins to noting the compositional biases or motifs which are obvious from manual inspection of the protein sequences. This method has the risk that important patterns in the data are missed and that rules are not applied consistently in identifying these patterns. Ideally the context of the proteome as a whole should also be taken into account. The more specific a feature is to the proteins of interest (POIs) the more likely it is to be involved in the specific function of those proteins. This notion is based on the wellestablished biological principle that the primary sequence of a protein is a strong determinant of molecular function, and that proteins with similar functions tend to share regions of sequence similarity. Thus, sequence motifs shared by a group of biomineral associated proteins are more likely to be involved in the specific function of those proteins if they are rare in the background proteome than if they are commonly found motifs. This principle is already used in various sequence analysis tools, including those seeking to identify important motifs (Wagih et al. 2016). Although there are many tools available that allow researchers to investigate the properties of protein sequences in silico, including analysis of compositional bias, sequence complexity, intrinsic disorder and sequence motifs, such tools are not always easy to use. Some require 
74

command line use, data input formats differ, some can only run on one protein at a time and most require post-processing of the output to format the data for statistical and graphical analyses in commonly used environments such as Microsoft ${ }^{\circledR}$ Excel ${ }^{\circledR}$ or R. These tools also rarely allow researchers to compare two sets of sequences.

Here we present ProminTools, a set of easy-to-use tools for the statistical comparison of two sets of protein sequences, available as apps in the CyVerse Discovery Environment (https://de.cyverse.org/) (Merchant et al. 2016) or to run locally from a Docker ${ }^{\mathrm{TM}}$ container. The inputs are simply two fasta files containing the proteins of interest (POIs) and the background proteome respectively, while the outputs include data tables and an html document containing graphical summaries of the data and interactive tables for data exploration. To demonstrate the utility of these tools, we reanalyse a published data set of shell matrix proteins (SMPs) from the giant limit Lottia gigantea (Mann \& Edsinger 2014).

\section{Materials and Methods}

\section{Promin Tools structure}

The inputs for ProminTools are two fasta formatted files: the first containing the protein set of interest (POI set), and the second the reference or background proteins (typically the predicted proteome of the organism of interest). The background proteins are used for statistical comparisons with the POI sets, allowing the user the answer the following question: 'Are the features observe in the POI common in the background sequences or are they unusual?'.

ProminTools has two component programmes: "Protein Motif Finder" and "Sequence Properties Analyzer”. Both are written in Perl and R and bundled with all dependencies in Docker ${ }^{\mathrm{TM}}$ (www.docker.com) containers. They can be run from Apps within the CyVerse Discovery Environment (Merchant et al. 2016) or on a personal computer via Docker ${ }^{\mathrm{TM}}$ Desktop. The 
97 primary outputs of the tools are data tables summarising key information from the comparison of

98 the two sequences sets. The tools use these tables to generate an html file with a graphical

100

101

102

103

104

105

106

107

108

109

110

111

112

113

114

115

116

117

118

119

summary of the information along with explanations, statistical analyses and interactive versions of certain data tables. A publication ready SVG (Scalable Vector Graphic) formatted figure is also generated by Protein Motif Finder. The R script that generates the html file from the data tables is also an output of the tools, allowing the user to reproduce the figures in the html report and to provide a starting point for further analyses specific to the data set. For licence information for all components of ProminTools the reader is referred to Data S1.

\section{Analyses performed by "Protein Motif Finder"}

\section{Motif finding with motif-x}

Protein Motif Finder uses the motif-x engine (Schwartz \& Gygi 2005; Wagih et al. 2016) for motif finding. This engine was chosen because it breaks sequences down into their constituent motifs, by an iterative procedure that avoids oversimplification of motifs and prioritises motifs that are most enriched relative to a background sequence set. It is exhaustive for a given p-value and generates definite motifs rather than a position weight matrix, which simplifies downstream analyses and is more useful to molecular biologists. In this work it was always run with the recommended, conservative, binomial $p$-value of $10^{-6}$, but this parameter is user customisable in Protein Motif Finder. The motif width is also user customisable, while the minimum occurrences parameter is hard coded at a value of five. Motif-x is run via the R module rmotif-x, centred on each amino acid sequentially and the results are combined. This procedure means that some motifs are likely to be redundant. For example, if the central residue is ' $\mathrm{S}$ ' and the motif width 7 then the motifs '...S.S..' and '..S.S... (where dot represents any amino acid) may both be identified, but these would be collapsed to the single motif ' $\mathrm{S} . \mathrm{S}$ '. Note that this procedure is 
120 conservative with respect to the original p-value calculated by motif-x. Significant motifs are

121 then enumerated in the POI and the background sequence sets, and motif counts and enrichments

122 reported in the output tables. Downstream analyses do not rely on the motif-x p-value, but only

123 on calculated enrichment values for the motifs.

\section{Graphical representations of motif data}

125 To provide a visual summary of the motif data, the motifs are represented in three wordclouds in the Protein Motif Finder output, which take into account two distinct measures of 'importance'.

127 The first is the number of proteins in which that motif is found. The more proteins containing the motif, the more likely it is to have general importance in the function of the group of proteins.

The second measure is the enrichment of the motif. The more enriched the motif the more unusual it is and thus is more likely to be involved in the specific function of these sets of proteins. A third measure attempts to combine the previous two by scaling them equivalently and then taking the product of the scaled values (PS-value). This measure prioritises motifs that are both highly enriched and found in a high proportion of the proteins.

134 In the output, proteins that are biased in sequence composition are also clustered based on their motif number and motif enrichment. The distance measure for clustering was calculated as one minus the Distance Correlation (Szekely et al. 2013) for all pairwise combinations of proteins or 137 motifs, since this method is especially robust to outliers and produces reasonable results across a variety of datasets. Hierarchical clustering was performed using the Ward.D method. In Heatmap

139 1, the following filters are applied to select proteins and motifs for clustering: 1) Select proteins that contain a biased region (fLPS p-value $<10^{-20}$; user adjustable), 2) Remove infinitely

141 enriched motifs. 3) Remove motifs not present in at least 3 POI proteins (if $>10$ proteins in POI 142 set). 4) Select the 70 overall most enriched motifs. 5) Select proteins with a good motif based 
143 correlation to at least one other protein (dcor $>0.65$, user adjustable). The filters for Heatmap 2

144 are the same except that filter 3 is not applied. The same protein and motif set is displayed in

145 Heatmap 3 as in Heatmap 2 except that motif count is displayed instead of motif enrichment.

146 Analyses performed by "Sequence Properties Analyzer"

147 Sequence Properties Analyzer performs the following analyses:

148 Amino acid enrichment

149 Compositional bias is analysed using fLPS (Harrison 2017) and the results collated to several

150 files described in the html output of the program.

151 Significance of sequence bias

152 To estimate the probability of obtaining the observed bias in amino acid composition in the POI

153 set by random sampling of the background proteome, the following procedure was implemented.

154 First the degree of bias was quantified by calculating a bias index (BI):

155

$$
B I=\sum_{\text {Amino acids }} \sqrt{(\text { POI freq. - Proteome freq. })^{2}}
$$

156

157

158

159

160

161

162

163

164

165

Where POI freq. is the frequency of the amino acid in the POI proteins, while Proteome freq. is the frequency of the amino acid in the background sequence set. The $B I$ is calculated for 1000 random samples of the background sequence set, each containing the same number of sequences as the POI set. A kernel density estimate of the distribution of $B I$ is calculated, and a function approximating this distribution is generated. The area under the curve greater than the $B I$ value of the POI set is used as an estimate of the probability of obtaining a sequence set of this degree of bias by chance, given this particular background proteome.

The only program we are aware of that makes a similar calculation is Composition Profiler (Vacic et al. 2007). However this makes the assumption that all POI sequences come from the same underlying distribution of amino acid frequencies and tests whether this distribution is 
166 significantly different from the background. ProminTools does not make this assumption, but

167 accepts that the POI set my contain proteins with different types of bias, and thus analyses bias

168 per se without reference to the type of bias.

169 Sequence complexity

170 The program SEG (Wootton \& Federhen 1993) is used to identify low complexity regions in the

171 datasets using default parameters, although these are customisable by the user in Sequence

172 Properties Analyzer. For each protein, the percentage of the sequence identified as low

173 complexity is calculated, and a Wilcoxon rank sum test with continuity correction is used to test

174 whether there is a significant difference in the distribution of this percentage length between the

175 POI and the background sequence set.

176 Intrinsic disorder

177 Predicted intrinsic disorder was calculated using the VSL2 predictor (Peng et al. 2006), due to its

178 speed and good accuracy (Nielsen \& Mulder 2019). This is the most time consuming step of

179 Protein Sequence Analyzer and is thus parallelized in the implementation. For each protein, the

180 percentage of the sequence identified as intrinsically disordered is calculated and a Wilcoxon

181 rank sum test with continuity correction is used to test whether there is a significant difference in

182 the distribution of this percentage length between the POI and the background sequence set.

183 Charged clusters

184 Clusters of charged amino acids are identified using the SAPS software (Brendel et al. 1992).

185 Data and methods for validation of Promin Tools

186 Representative $\mathrm{CxxC} \mathrm{Zn}$ finger proteins were chosen from the Wingender database (Wingender

187 et al. 2013) and compared to the human proteome Swissprot database accessed on the 28/05/20.

188 For the analysis of human low complexity proteins, all models were downloaded from Ensemble 
189

190

191

192

193

194

195

196

197

198

199

200

201

202

203

204

205

206

207

208

209

210

211

version 100. Models shorter than 100 amino acids were removed, as were models with internal stop codons, resulting in 89562 proteins that were used as the background sequence in the

analysis. The foreground sequence set was the 500 most biased proteins identified using fLPS (Harrison et al. 2017). These proteins were annotated using eggNOG mapper (Huerta-Cepas et al. 2017, 2019) with parameters "taxonomic scope hominidae, -target_orthologs all -seed_ortholog_evalue 0.001 --seed_ortholog_score 60 --query-cover 20 ”.

\section{L. gigantea shell matrix proteome data}

To illustrate the utility of the ProminTools package, we used the shell matrix proteome of the giant limpet, L. gigantea as published by Mann and Edsinger (2014) which is a reanalysis of their original data (Mann et al. 2012). The protein identifiers were extracted from table S1 of (Mann \& Edsinger 2014) and the protein sequences extracted from files Lotgi1_GeneModels_AllModels_20070424_aa.fasta and Lotgi1_GeneModels_FilteredModels1_aa.fasta which were downloaded from the JGI (https://mycocosm.jgi.doe.gov/Lotgi1/Lotgi1.home.html) on the 5/02/2020. The final set of proteins consisted of 381 sequences, and are available in Data $S 2$. This is one less than the number of accepted identifications in (Mann \& Edsinger 2014) since the protein Lotgi|172500 was not available in any database.

\section{Analyses of $L$. gigantea data using ProminTools}

ProminTools was run locally using the shell matrix proteins as the foreground sequences and the 'Lotgi1_GeneModels_FilteredModels1_aa.fasta' file as the background proteome. The filtered models were chosen as they were considered likely to be a closer representation of the true proteome of L. gigantea than the 'All Models' set, and thus the more appropriate set for statistical comparisons. 


\section{Additional analyses}

213 Proteins were clustered based on motif content as an output of the Protein Motif Finder tool. To

214 determine an optimal cluster number, manual inspection of a plot of the cindex (Hubert \& Levin

215 1976) for cluster sizes 2 - 50 was carried out. A cluster number of 8 seemed appropriate for the

216 present work, since it captured the major patterns in the data without becoming too granular.

217 These clusters were the input for further runs of Protein Motif Finder.

218 Sequence similarity was quantified using and all vs all pairwise BLASTp analysis, reporting the 219 percentage identity of the top scoring high scoring pair (HSP), after applying an e-value cut-off 220 of 0.01 and a cut-off specifying that the HSP alignment length must be at least $20 \%$ of the query 221 length.

\section{Results}

Promin Tools provides a user-friendly method to analyse biomineralization proteomes

224 The Docker ${ }^{\mathrm{TM}}$ image containing ProminTools can be run via a GUI on the CyVerse Discovery 225 Environment without any need for use of the command line. Runtimes on CyVerse are variable due to variable resource availability, but a typical analysis with either Protein Motif Finder or

227 Sequence Properties Analyzer takes between 30 and 120 minutes to complete. Although

228 ProminTools is designed to run in a Unix environment, it can also be run on a windows PC via

229 Docker $^{\mathrm{TM}}$ Desktop with simple commands in Windows ${ }^{\circledR}$ Power Shell ${ }^{\mathrm{TM}}$ (for details see 230 https://github.com/skeffington/Promin-tools). On a Window ${ }^{\circledR} 10$ machine with an Intel® Core ${ }^{\mathrm{TM}}$ 231 i7-2600 3.4 GHz processer and 16 GB RAM, Protein Motif Finder completed analysing the $L$. 232 gigantea data set in 11 min 30 s provided with 1 core and 2.5 GB RAM, while the Sequence 233 Properties Analyser completed in $32 \mathrm{~min} 2 \mathrm{~s}$ provided with 5 cores and 5 GB RAM. The 234 ProminTools workflow is summarised in Fig. 1. 


\section{Validation of Promin Tools}

\section{Suitability for a range of data inputs}

237 To ensure stability and good performance, we have tested ProminTools on a number of

238 published and unpublished biomineralization datasets and used synthetic data to ensure that the

239 program deals sensibly with unusual situations, such as small numbers of POI sequences or no

240 motifs being found. An example analysis of a second data set, of proteins from freshwater

241 mussel shells (Marie et al. 2017), is provided in Data S3.

\section{Validation with negative control protein sets}

243 Five sets of 100 proteins were drawn at random from the L. gigantea proteome and each used as

244 the POI set to run ProminTools in five separate analyses. No enriched motifs were reported in

245 any of the analyses. In all analysis, there were no significant differences in the degree of

246 sequence bias, sequence complexity or intrinsic disorder between the random 'POI' set and the

247 background proteome. Representative analyses are provided in Data S4.

248 Validating motif retrieval in Protein Motif Finder

249 Motif finding in ProminTools relies on the motif-x motif finding engine, which has already been

250 well validated (Schwartz and Gygi 2005). However to ensure that there were no bugs in our

251 post-processing of the output we spiked motifs at known frequencies into a set of protein

252 sequences and ran Protein Motif Finder with these sequences as foreground, and the un-spiked

253 sequences as background. The spiked motifs were recovered at the expected frequencies.

254 We also validated Protein Motif Finder on a groups of sequences containing motifs that have

255 already been established as important for protein function. For example CXXC class zinc finger

256 factors are transcription factors and histone methyltransferases that bind to CpG elements via

257 zinc fingers. The $\mathrm{Zn}$ binding residues consist of cysteines arranged in $\mathrm{CGxCxxC}$ motifs 
258 (Wingender et al. 2013). Using a set of CXXC zinc fingers factors as the POI set and the human

259 proteome as the background set, Protein Motif Finder correctly identified CG.C..C as the most

260 important motif, and found it to be 194 fold enriched in these proteins relative to the background

261 proteome (Data S5).

262 It should be noted that not all motifs important for a protein's function will be enriched relative

263 to the background. For example the L..LL motif is important in protein-protein interactions that

264 regulate transcription (Plevin et al. 2005). Using the 55 Swissprot proteins annotated as

265 possessing an L..LL motif as the POI set and the Swissprot human proteome as background,

266 Protein Motif Finder does not recover the L..LL motif (Data S6). This is because L..LL is

267 relatively common in other contexts, and so is not significantly enriched in the POI set.

268 Validating the biological meaning of clustering by motif enrichment

269 A key output of Protein Motif Finder is clustering of the POIs based on their motif enrichment. The

270 usefulness of this clustering is based on the assumption that proteins within a cluster are likely to be

271 involved in similar molecular processes. To test this assumption on a well annotated proteome, but

272 focusing on biased sequences similar to those expected from biomineral associated proteins, we analysed

273 the 500 most biased sequences from the human proteome. Of these, 303 could be annotated by eggNOG

274 mapper (Huerta-Cepas et al. 2017) and they fell into 120 clusters when analysed with Protein Motif

275 Finder (Data S7). Remarkably, proteins within a cluster all shared the same eggNOG functional

276 annotation in 117 of the clusters, even when the proteins diverged significantly in primary sequence

277 similarity as assessed by global alignments (Table 1). Of the input proteins, 53 were collagens, and 11

278 different types of collagen were successfully separated into separate clusters. The members of three

279 clusters mapping to more than one annotation were clearly related within a cluster: two clusters contained

280 two different types of collagen, while one cluster contained two types of epidermal growth factor-like

281 domains. 
282 Global properties of $L$. gigantea shell matrix proteome

283 Previous analyses of the L. gigantea shell matrix proteome (Mann et al. 2012; Mann \& Edsinger

284 2014; Marie et al. 2013a) had noted a tendency for the proteins to be low complexity and

285 disordered and that some proteins were enriched in particular residues. Here, ProminTools was

286 used to put these observations on a more quantitative footing (Data 58, Data $S 9$ ) and to discover

287 enriched sequenced motifs in the data set from Mann et al. (2014), which contained 381 proteins.

$288 \mathrm{G}$ and P rich motifs were found to be enriched most frequently among the proteins (Fig. 2A).

289 Given that we are seeking to find the motifs that are shared within a group of proteins, Protein

290 Motif Finder excludes motifs found in fewer than four proteins from certain plots to prevent the

291 picture being dominated by a highly enriched motif found in very few proteins. The result can be

292 seen in Fig. 2B, where Q containing motifs displayed the greatest enrichments relative to the

293 background proteome. For example QQP was enriched 7.5 fold while Q.N.Q was enriched 6.1

294 fold (see data tables in Data $S 8$ for these numbers). In general there is often a negative

295 correlation between the number of proteins in a set containing motifs and the enrichment of those

296 motifs. These two measures are combined (see Materials and Methods) in Fig. 2C, which

297 emphasises motifs found in a high number of proteins with high enrichment (a high PS-value).

298 For example, GG is found in 270 proteins and is 1.8 fold enriched, G..D in 234 proteins at 1.4

299 fold enrichment and NG in 249 proteins at 1.53 fold enrichment (Data S8).

300 The analysis of sequence bias and amino composition bias with Sequence Properties Analyzer

301 was concordant with the motif finding results, in that G, P, Q and A were the most enriched

302 amino acids (Fig. 2D). H, I, K, L, W, E and F were found to be the most depleted relative to the

303 background proteome. The most commonly enriched amino acids were P, G, A and C (enriched

304 in 87, 82, 74 and 69 proteins respectively, Fig. 2E). Amino acid residues $\mathrm{C}$ and A are not found 
305

306

307

308

309

310

311

312

313

314

315

316

317

318

319

320

321

322

323

324

325

326

among the most enriched motifs or the motifs with the highest PS-value, indicating that the

proteins are sometimes enriched in an amino acid without that amino acid being embedded in a

particular primary sequence context.

The shell matrix proteins showed a clear tendency to contain more low complexity sequence than the background proteome (Wilcoxon rank sum test, $p=7.6 \times 10^{-6}$, Fig. $2 F$ ) but there was no significant tendency for the sequences to contain a greater proportion of predicted disordered sequence than the background proteome $(p=0.1)$. The shell matrix protein set contained a similar proportion of proteins with negative and positive clusters of amino acids to the background proteome (Fig. 2H).

\section{Clustering of proteins based on motif content reveals relationships not found by blast} searches

The Sequence Properties Analyzer carries out three hierarchical clustering analyses (Data S8, Materials and Methods). Eight protein clusters were identified (Fig. 3A), six of which contained more than two proteins. To investigate the nature of each cluster, Protein Motif Finder was rerun on each of the six main clusters (Data S10). Clusters 1 and 2 were riche in G containing motifs, especially NG.GG in cluster 1. Cluster 3 contained proteins rich in D containing motifs (especially D.NDD); cluster 4 in a variety of Q containing motifs; cluster 5 in C.I.P.D and C..YC..G and cluster 6 in various T and P containing motifs. By analysing the specific set of proteins in each cluster, the motifs identified are more specific to those proteins, and thus differ from the most enriched motifs in the data set as a whole displayed in Fig. 3A. For example D.NDD is the most prominent motif from the reanalysis of cluster 3, but is not among the most 
327 enriched motifs in the global analysis of the entire data set, demonstrating the value of this

328 iterative approach.

329 Given the results of our validation analysis with human low complexity proteins, it can be

330 hypothesised that proteins found within the same cluster have related function. Only one of the

331 proteins (Lotgi1|143247, cluster 5) has an annotation: a 'four disulphide core domain protein'

332 (Pfam PF00095), suggesting that it may function as a protease inhibitor. Given the lack of

333 annotations, it was not possible to further test the relationship between cluster membership and

334 function using the L. gigantea data.

335 We next asked whether the motif clusters reflected larger scale sequence similarity between the

336 proteins within a cluster. To this end, protein sequences in each cluster were subject to an all-vs-

337 all pairwise BLASTp analysis, which is summarised in the matrices in Fig. $3 C$ for six of the

338 clusters. In general larger scale sequence similarity was low within clusters, with only three

339 protein pairs from the five clusters displaying identity above $50 \%$ for the highest scoring HSP.

340 This demonstrates that the clustering method can be used to find similarities that are not obvious

341 from BLAST searches.

342 Discussion

343 Proteins are a prominent part of the organic matrices of many biominerals and are thought to

344 have a number of roles including catalysis, templating, and control of nucleation and crystal

345 growth. Studies of biomineral associated proteins understandably often emphasise proteins with

346 conserved domains, which lend themselves to discussions of their possible molecular functions.

347 However most studies also identify many proteins of unknown function, many of which appear

348 to be low complexity in nature, with biased compositions and a high proportion of intrinsic

349 disorder. Although authors often carefully inspect their protein sequences and note sequences 
350 that appear particularly rich in certain residues or motifs, and note the degree of disorder, this

351 information is rarely put in the context of the predicted proteome as a whole.

352 Here we introduce ProminTools, a user friendly package that allows researchers to glean more

353 information from primary sequences of proteins of unknown function and put this in the context

354 of the background proteome. Importantly, ProminTools allows users with minimal bioinformatic

355 skills to run a suite of analyses and produce visualization that would otherwise require a lot of

356 scripting. The giant limpet $L$. gigantea has a complex shell matrix proteome for which two

357 different data sets exist. The data analysed in the present study derives from all shell layers

358 (Mann et al. 2012, Mann \& Edsinger 2014), and is thus more complex than the second data set

359 that is derived from the aragonite shell layers only, excluding the calcitic layers (Marie et al.

360 2013a).

361 ProminTools revealed a complex array of strongly enriched motifs in the Mann et al. (2014) data

362 set, which were not uncovered in the original study. Q, P and G rich motifs were particularly

363 prominent and the proteins could be clustered based on their motif content even when they

364 shared little larger scale sequence similarity. Re-running Protein Motif Finder on each of these

365 clusters revealed unique motif profiles that could be hypothesised to be important for the

366 molecular function of proteins in the group. For example, one group was enriched in acidic (D

367 rich) motifs, another in $\mathrm{Q}$ and $\mathrm{P}$ rich motifs and other in $\mathrm{G}$ rich motifs. Interestingly, the Marie

368 et al. study also identify a group of low complexity proteins rich in Q, suggesting that the

369 functions of these proteins could be important for formation of all shell layers or just the

370 aragonite layers, but that they are unlikely to be specific to the calcite layers.

371 We hypothesise that clustering protein sequences with biased composition based on their motif

372 enrichment patterns can be used to group proteins of related function. Although this hypothesis 
373 has yet to be confirmed on biomineral associated protein data sets, we show that this procedure

374 can group functionally related proteins of biased composition from humans. Additional support

375 for the idea comes from a previous study in which accurate predictors of enzyme function were

376 built using the motif content of protein sequences (Ben-Hur et al. 2006).

377 We found that the shell matrix proteins as a group were significantly lower in complexity than

378 the background proteome, providing a statistical underpinning for this observation, and

379 supporting the conclusion of Marie et al. (2013a) who noted the high proportion of low

380 complexity sequences in their data set. The Mann et al. studies (Mann et al. 2012; Mann \&

381 Edsinger 2014) highlight several proteins in their data which have high degrees of intrinsic

382 disorder. Here, using the Sequence Properties Analyzer we were able to demonstrate that this is

383 not a general feature of the data set, which is not predicted to be significantly more disordered

384 than the background proteome. This highlights the importance of the proteome context when

385 assigning significance to protein features, and demonstrates that the generally observed

386 correlation between protein disorder and low complexity (Mier et al. 2019) does not hold in

387 every data set.

388 The role of low complexity regions in biomineralization has only been determined in a very few

389 cases. For example, the enamel protein Amelogenin has a central block of hydrophobic sequence

390 rich in $\mathrm{P}, \mathrm{H}$ and $\mathrm{Q}$. Intramolecular hydrophobic interactions involving this regions are thought to

391 be critical for self-assembly of Amelogenin into nanospheres and higher order structures that

392 regulated crystal growth (Wang \& Nilsen-Hamilton 2012). It is possible that the Q and P rich

393 regions in the L. gigantea shell matrix proteins might have a similar role in driving self-assembly

394 processes. 
395 Although at present we can only speculate on the role of low complexity proteins in

396 biomineralization, it is clear that low complexity sequences are not unique to biomineralization

397 related proteins. Depending on the species, $22-36 \%$ of residues in eukaryotic proteins fall into

398 low complexity regions (Wootton 1994). It remains to be investigated whether the low

399 complexity regions of biomineralization related proteins have features that set them apart from

400 other low complexity regions in proteomes, and ProminTools could be used to investigate such

401 questions.

402 ProminTools allows researchers to easily find patterns in their data, but it has limitations and

403 judgement should be applied in interpreting the output. For example, patterns found by

404 ProminTools can reflect technical biases as well as biological signals. Post-translational

405 modifications of particular residues could affect peptide detectability and thus protein inference,

406 leading to biases in the input data. It should also be remembered that ProminTools is primarily a

407 tool for hypothesis generation. For example, proteins which share similar motifs can be

408 hypothesised to perform similar molecular functions, but this may or may not be the case for a

409 particular biological system, and experimental validation is required. ProminTools will be at its

410 most useful when combined with other methods for spotting repeating patterns in sequences (e.g.

411 HhpreID (Zimmermann et al. 2018), Meme (Bailey et al. 2009) or simply inspecting dot-plots)

412 and when put in the context of additional information such as known domain content, post-

413 translational modifications, phylogenetic distributions and expression patterns.

414 We would like to point out that ProminTools can be used for any pairwise comparison of sets of

415 protein sequences. For examples, protein sets associated with different part of a biomineral or

416 different developmental stages could also be compared, and if carefully carried out, cross-species

417 comparisons could also be made. The latter could be particularly useful, since the fast evolving 
418 nature of low complexity sequences (McDougall et al. 2013) can make it difficult to detect

419 homology. It could also be applied to other protein sets rich in low complexity sequences, such

420 as proteins found in pathological amyloids associated with diseases such as Alzheimer's and

421 Parkinson's (Kumari et al. 2018).

\section{Conclusions}

423 ProminTools will help researchers generate new hypotheses about the important of particular

424 motifs and protein chemistries in their system of interest and provide new directions for

425 experimental work. Putting the patterns identified into the context of the rest of the proteome

426 ensures that features that are genuinely overrepresented in the POIs are prioritised for further

427 study.

\section{Acknowledgements}

429 We gratefully acknowledge Dr. André Scheffel for advice and critical reading of the manuscript.

\section{Author contributions}

431 AS conceived the study, wrote the tools, compiled the figures and wrote the manuscript. AD

432 developed the container structure and reviewed the manuscript.

\section{Software availability}

434 ProminTools is available in two apps with graphical user interfaces from the Cyverse Discovery

435 Environment (https://de.cyverse.org/). Users need to create an account, but then have access to a

436 large number of tools and high performance computing. Docker images are most easily accessed

437 from Docker Hub (https://hub.docker.com/repositories/biologistatsea/), but the user must have a

438 (free) account. The images can also be downloaded from FigShare (Data S11, Sequence 
439 Properties Analyzer: https://doi.org/10.6084/m9.figshare.12670817.v1 Protein Motif Finder:

440 https://doi.org/10.6084/m9.figshare.12667070.v1) and detailed and accessible instructions on 441 usage are available at https://github.com/skeffington/Promin-tools.

\section{References}

443 Bailey TL, Boden M, Buske FA, Frith M, Grant CE, Clementi L, Ren JY, Li WW, and Noble

444

445

446

447

448

449

450

451

452

453

454

455

456

457

458

459

460

461
WS. 2009. MEME SUITE: tools for motif discovery and searching. Nucleic Acids Research 37:W202-W208. 10.1093/nar/gkp335

Ben-Hur A, Brutlag D. 2006. Sequence motifs: highly predictive features of protein function. In: Guyon I, Nikravesh M, Gunn S, Zadeh L, eds. Feature Extraction. 625-645 Berlin, Heidelberg: Springer.

Brendel V, Bucher P, Nourbakhsh IR, Blaisdell BE, and Karlin S. 1992. Methods and algorithms for statistical analysis of protein sequences. Proc Natl Acad Sci U S A 89:2002-2006. 10.1073/pnas.89.6.2002

Evans JS. 2019a. The biomineralization proteome: Protein complexity for a complex bioceramic assembly process. Proteomics 19. 10.1002/pmic.201900036

Evans JS. 2019b. Composite materials design: Biomineralization proteins and the guided assembly and organization of biomineral nanoparticles. Materials 12 . $10.3390 /$ ma12040581

Harrison PM. 2017. fLPS: Fast discovery of compositional biases for the protein universe. Bmc Bioinformatics 18. 10.1186/s12859-017-1906-3

Huerta-Cepas J, Forslund K, Pedro Coelho L, Szklarczyk D, Jensen JL, von Mering C, Bork P. 2017. Fast genome-wide functional annotation through orthology assignment by eggNOG-mapper. Molecular Biology and Evolution 34:2115-2122.

Peer] reviewing PDF | (2020:04:47682:1:1:NEW 18 Jul 2020) 
462 Huerta-Cepas J, Szklarczyk D, Heller D, Hernández-Plaza A, Forslund SK, Cook H, Mende D, 463 Letunic I, Rattei T, Jensen LJ, von Mering C, Bork P. 2019. eggNOG 5.0: a hierarchical,

464

465

466

467

468

469

470

471

472

473

474

475

476

477

478

479

480

481

482 functionally and phylogenetically annotated orthology resource based on 5090 organisms and 2502 viruses. Nucleic Acids Res. 47: D309-D314. doi: 10.1093/nar/gky1085

Hubert LJ, and Levin JR. 1976. A general statistical framework for assessing categorical clustering in free recall. Psychological Bulletin 83:1072-1080. 10.1037/00332909.83.6.1072

Jackson DJ, Mann K, Haussermann V, Schilhabel MB, Luter C, Griesshaber E, Schmahl W, and Worheide G. 2015. The Magellania venosa biomineralizing proteome: A window into brachiopod shell evolution. Genome Biology and Evolution 7:1349-1362. $10.1093 / \mathrm{gbe} / \mathrm{evv} 074$

Kotzsch A, Pawolski D, Milentyev A, Shevchenko A, Scheffel A, Poulsen N, Shevchenko A, and Kroger N. 2016. Biochemical composition and assembly of biosilica-associated insoluble organic matrices from the Diatom Thalassiosira pseudonana. Journal of Biological Chemistry 291:4982-4997. 10.1074/jbc.M115.706440

Kumari B, Kumar R, Chauhan V, and Kumar M. 2018. Comparative functional analysis of proteins containing low-complexity predicted amyloid regions. PeerJ 6. $10.7717 /$ peerj. 5823

Le Roy N, Jackson DJ, Marie B, Ramos-Silva P, and Marin F. 2014. The evolution of metazoan alpha-carbonic anhydrases and their roles in calcium carbonate biomineralization. Frontiers in Zoology 11. 10.1186/s12983-014-0075-8

Peer) reviewing PDF | (2020:04:47682:1:1:NEW 18 Jul 2020) 
483 Mann K, Edsinger-Gonzales E, and Mann M. 2012. In-depth proteomic analysis of a mollusc 484 shell: acid-soluble and acid-insoluble matrix of the limpet Lottia gigantea. Proteome Science 10. 10.1186/1477-5956-10-28

486

487

488

489

490

491

492

493

494

495

496

497

498

499

500

501

502

503

Mann K, and Edsinger E. 2014. The Lottia gigantea shell matrix proteome: re-analysis including MaxQuant iBAQ quantitation and phosphoproteome analysis. Proteome Science 12. Artn 28

$10.1186 / 1477-5956-12-28$

Mann K, Macek B, and Olsen JV. 2006. Proteomic analysis of the acid-soluble organic matrix of the chicken calcified eggshell layer. Proteomics 6:3801-3810. 10.1002/pmic.200600120

Marie B, Jackson DJ, Ramos-Silva P, Zanella-Cleon I, Guichard N, and Marin F. 2013a. The shell-forming proteome of Lottia gigantea reveals both deep conservations and lineagespecific novelties. Febs Journal 280:214-232. 10.1111/febs.12062

Marie B, Ramos-Silva P, Marin F, and Marie A. 2013b. Proteomics of $\mathrm{CaCO}_{3}$ biomineralassociated proteins: How to properly address their analysis. Proteomics 13:3109-3116. 10.1002/pmic. 201300162

Marie B, Arivalagan J, Mathéron L, Bolbach G, Berland S, Marie A, Marin F. 2017. Deep conservation of bivalve nacre proteins highlighted by shell matrix proteomics of the Unionoida Elliptio complanata and Villosa lienosa . J R Soc Interface 14:pii20160846

McDougall C, Aguilera F, and Degnan BM. 2013. Rapid evolution of pearl oyster shell matrix proteins with repetitive, low-complexity domains. Journal of the Royal Society Interface 10. $10.1098 /$ rsif.2013.0041 
504 Merchant N, Lyons E, Goff S, Vaughn M, Ware D, Micklos D, and Antin P. 2016. The iPlant 505 Collaborative: Cyberinfrastructure for enabling data to discovery for the life sciences. $506 \quad$ Plos Biology 14. 10.1371/journal.pbio.1002342

507 Mier P, Paladin L, Tamana S, Petrosian S, Hajdu-Soltesz B, Urbanek A, Gruca A, Plewczynski

508

509

510

511

512

513

514

515

516

517

518

519

520

521

522

523

524

525

526
D, Grynberg M, Bernado P, Gaspari Z, Ouzounis CA, Promponas VJ, Kajava AV, Hancock JM, Tosatto SCE, Dosztanyi Z, and Andrade-Navarro MA. 2019. Disentangling the complexity of low complexity proteins. Brief Bioinform. 10.1093/bib/bbz007

Nielsen JT, and Mulder FAA. 2019. Quality and bias of protein disorder predictors. Scientific Reports 9. 10.1038/s41598-019-41644-w

Peng K, Radivojac P, Vucetic S, Dunker AK, and Obradovic Z. 2006. Length-dependent prediction of protein intrinsic disorder. Bmc Bioinformatics 7. 10.1186/1471-2105-7-208

Plevin MJ, Mills MM, Ikura M. 2005. The LxxLL motif: a multifunctional binding sequence in transcriptional regulation. Trends Biochem Sci. 30:66-69. doi: 10.1016/j.tibs.2004.12.001

Schwartz D, and Gygi SP. 2005. An iterative statistical approach to the identification of protein phosphorylation motifs from large-scale data sets. Nature Biotechnology 23:1391-1398. $10.1038 / \mathrm{nbt} 1146$

Skinner HCW, and Jahren AH. 2007. Biomineralization. Treatise on Geochemistry, 1-69.

Song XR, Liu ZQ, Wang LL, and Song LS. 2019. Recent advances of shell matrix proteins and cellular orchestration in marine molluscan shell biomineralization. Frontiers in Marine Science 6. 10.3389/fmars.2019.00041

Suzuki M, Saruwatari K, Kogure T, Yamamoto Y, Nishimura Y, Kato T, Nagasawa H. 2009. An Acidic matrix protein, Pif, is a key macromolecule for nacre formation. Science 325: 1388-1390.

Peer) reviewing PDF | (2020:04:47682:1:1:NEW 18 Jul 2020) 
527 Szekely, Gabor \& Rizzo, Maria. (2013). Partial distance correlation with methods for

528 dissimilarities. The Annals of Statistics. 42. 10.1214/14-AOS1255.

529 Wagih O, Sugiyama N, Ishihama Y, and Beltrao P. 2016. Uncovering phosphorylation-based

530 specificities through functional interaction networks. Molecular \& Cellular Proteomics

$531 \quad 15: 236-245.10 .1074 /$ mcp.M115.052357

532 Wang L, and Nilsen-Hamilton M. 2012. Biomineralization proteins: from vertebrates to bacteria. $533 \quad$ Frontiers in Biology 8:234-246. 10.1007/s11515-012-1205-3

534 Wingender E, Schoeps T and Dönitz J. TFClass: An expandable hierarchical classification of

535 human transcription factors. Nucleic Acids Res. 41, D165-D170 (2013). doi:

$536 \quad 10.1093 /$ nar/gks1123

537 Wootton JC. 1994. Non-globular domains in protein sequences: automated segmentation using 538 complexity measures. Computers \& Chemistry 18:269-285. 10.1016/0097$539 \quad 8485(94) 85023-2$

540 Wootton JC, and Federhen S. 1993. Statistics of local complexity in amino-acid-sequences and 541 sequence databases. Computers \& Chemistry 17:149-163. Doi 10.1016/0097-

$542 \quad 8485(93) 85006-X$

543 Zimmermann L, Stephens A, Nam SZ, Rau D, Kubler J, Lozajic M, Gabler F, Soding J, Lupas

544 AN, and Alva V. 2018. A completely reimplemented MPI bioinformatics toolkit with a 545 new HHpred server at its core. Journal of Molecular Biology 430:2237-2243.

546 10.1016/j.jmb.2017.12.007

547 


\section{Table $\mathbf{1}$ (on next page)}

Annotations of the 10 largest clusters identified by ProminTools in an analysis of human proteins of biased sequence composition 
2 Table 1:

3 Annotations of the 10 largest clusters identified by Promin Tools in an analysis of human

4 proteins of biased sequence composition.

\begin{tabular}{|l|l|l|l|l|}
\hline $\begin{array}{l}\text { Cluster } \\
\text { ID }\end{array}$ & $\begin{array}{l}\text { Cluster } \\
\text { size }\end{array}$ & $\begin{array}{l}\text { Identity } \mathbf{( \% )} \text { in all- } \\
\text { vs-all alignments } \\
\text { (min } ; \text { mean } ; \text { max })\end{array}$ & $\begin{array}{l}\text { eggNog annotation } \\
\text { carrying this } \\
\text { annotation (\%) }\end{array}$ \\
\hline 1 & 21 & $68.9 ; 87.7 ; 100$ & $\begin{array}{l}\text { Extracellular domain of unknown } \\
\text { function in nidogen (entactin) and } \\
\text { hypothetical proteins }\end{array}$ & 100 \\
\hline 2 & 19 & $93.4 ; 96.9 ; 100$ & Collagen type XI alpha 2 & 100 \\
\hline 3 & 14 & $99.8 ; 99.9 ; 100$ & Coiled-coil 2A & 100 \\
\hline 4 & 13 & $76.2 ; 88.1 ; 100$ & $\begin{array}{l}\text { Collagen triple helix repeat }(20 \\
\text { copies) }\end{array}$ & 100 \\
\hline 30 & 5 & $99.6 ; 99.7 ; 100$ & Nebulin repeat & 100 \\
\hline 33 & 5 & $78.0 ; 86.6 ; 99.5$ & Titin Z & 100 \\
\hline 39 & 5 & $57.4 ; 74.5 ; 100$ & $\begin{array}{l}\text { Neuroblastoma breakpoint family } \\
\text { member }\end{array}$ & 100 \\
\hline 40 & 5 & $94.6 ; 97.8 ; 100$ & $\begin{array}{l}\text { WAS WASL interacting protein } \\
\text { family member 1 }\end{array}$ & 100 \\
\hline 41 & 5 & $67.9 ; 80.5 ; 95.9$ & Golgin subfamily A member & 100 \\
\hline 114 & 5 & $99.8 ; 99.9 ; 100$ & $\begin{array}{l}\text { Neurogenic locus notch homolog } \\
\text { protein 4 }\end{array}$ & 100 \\
\hline
\end{tabular}




\section{Figure 1}

\section{Summary of ProminTools}

Proteomic datasets derived from analysis of biomineralizing organisms result in a fasta file containg the POI set which, along with the fasta file of the background proteome, make up the inputs for the ProminTools apps. The graphical interface shown is from the Cyverse Discovery Envronment (reproduced with permission). The outputs of the tools are detailed at the bottom of the figure. Attributions for the biomineral images are as follows: top left Vittina waigiensis by H. Zell (licence: CC BY-SA 3.0), top right - Radiolarian skeleton by Hannes Grobe (licence: CC BY 3.0), bottom left - Coccolithus pelagicus by Richard Lampitt and Jeremy Young, The Natural History Museum, London (licence: CC BY 2.5), bottom right Lottia mesoleuca by H. Zell (licence: CC BY-SA 3.0).

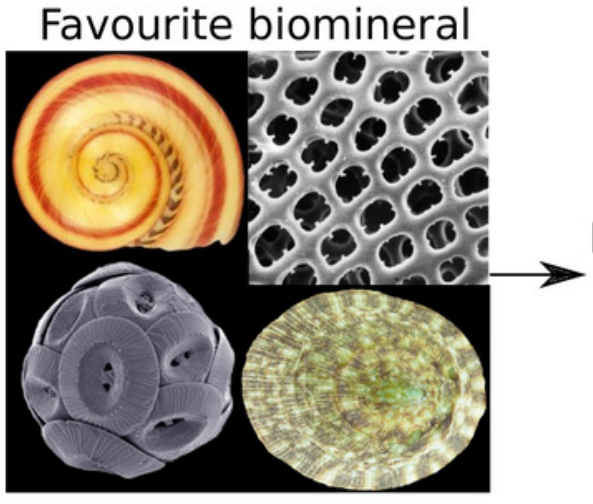

\section{Outputs:}

- html file

- Explanations of data tables

- Graphical summaries

- Interactive data tables

- Data tables

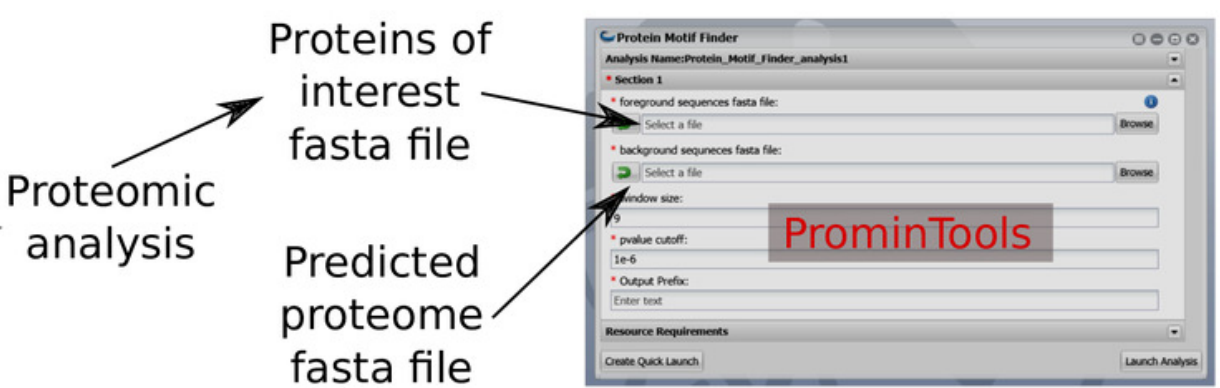

- Key figure as svg file

- R-script responsible for html report generation from the data tables<smiles>[V][V]</smiles>

- Reproduce and modify figures

- Further user-driven anlayses 


\section{Figure 2}

Properties of $L$. gigantea shell associated soluble proteome revealed by ProminTools.

Wordclouds are displayed where the height of the letter is proportional to: $\mathbf{A}$ the number of proteins in the SMP set containing the motifs, $\mathbf{B}$ the enrichment of the motif relative to the background proteome, and $\mathbf{C}$ the product of protein number and enrichment after scaling (PS-value). D The enrichment of amino acids in the SMP set relative to the background proteome. Values above zero indicate enrichment, and values below zero depletion. E The number of proteins in the SMP set enriched in each amino acid. Insert is a wordcloud summarizing the same data. $\mathbf{F}$ Density plot showing the distribution of the proportion of sequence length that is low complexity for the SMP proteins (labelled POI for Proteins Of Interest) and the background proteome. G Density plot showing the distribution of the proportion of sequence length that is predicted to be intrinsically disordered for the SMP proteins (POI) and the background proteome. $\mathbf{H}$ The proportion of sequences containing negatively and positively charged clusters of amino acids in the SMP proteins (POI) and the background proteome. 
A $\begin{gathered}\text { Number of proteins } \\ \text { containing motif }\end{gathered}$

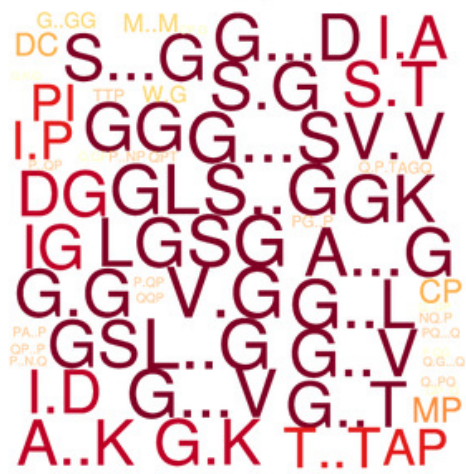

D

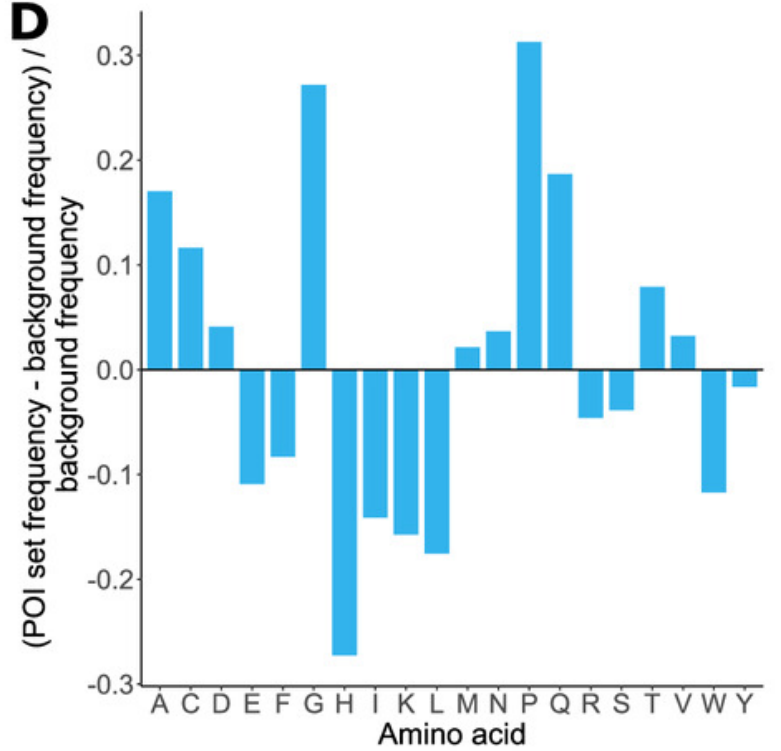

$\mathbf{F}$

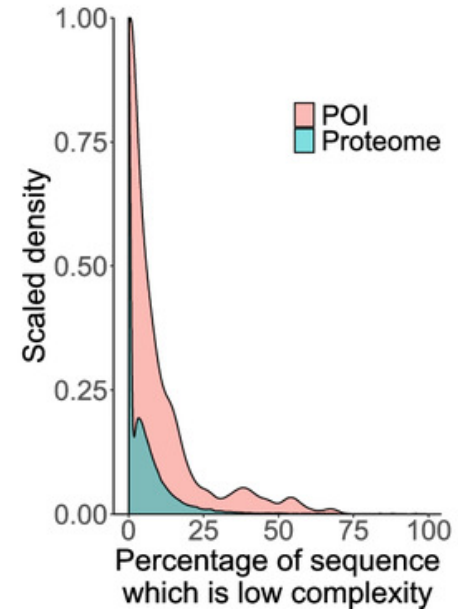

B

Enrichment

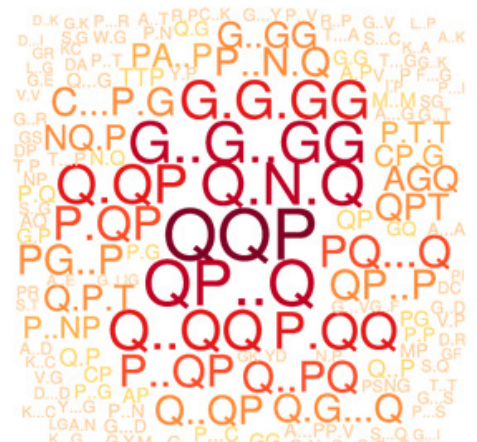

E

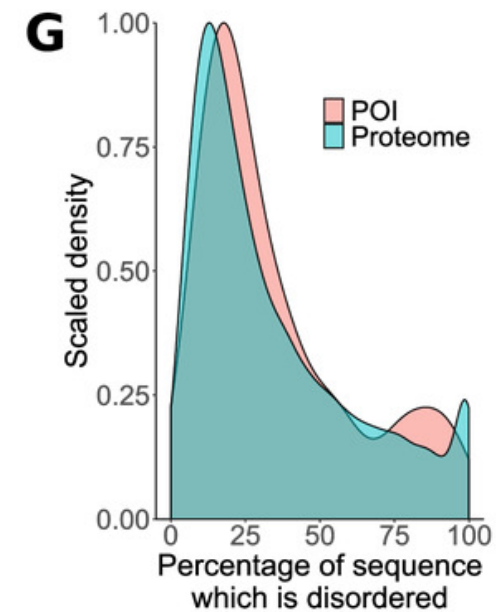

C Product of scaled

RGG...SS.GN.G T.ANGQ.PG..T IGG...VQ P G.P Q.GPGQPSG $L G$ A...GGGV.GDA GQ G.GP.GAP QQP G..DA.PDG A..T G.NP.Q GS AQ N.QA...AP.V
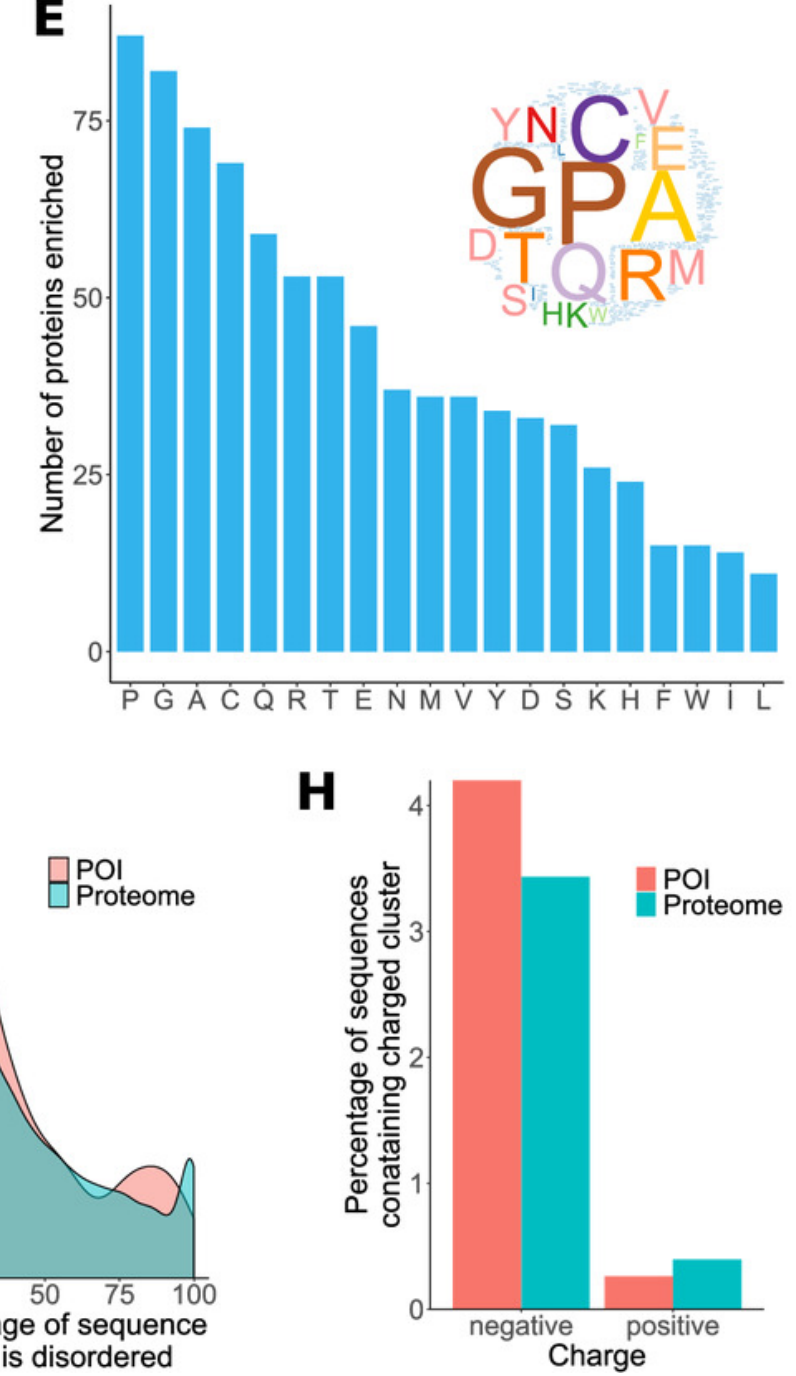


\section{Figure 3}

L. gigantea shell matrix proteins can be clustered based on motif content despite low sequence identity.

Figure 3: L. gigantea shell matrix proteins with biased composition can be clustered based on motif content despite low sequence identity. The heatmap displays (A) motif enrichment in the SMP set relative to the background proteome. Proteins are clustered by their motif enrichment pattern and motifs are clustered by their distribution amongst the proteins. Each motif is a row in the heatmap and each protein is a vertical column. For clusters1 - 6, a wordcloud (B) representing the PS-value of the enriched motifs are displayed in addition to a heatmap $(\mathbf{C})$ representing the percentage identity between all pairs of proteins in the cluster in an all-vs-all blastp analysis (see Materials and Methods). 


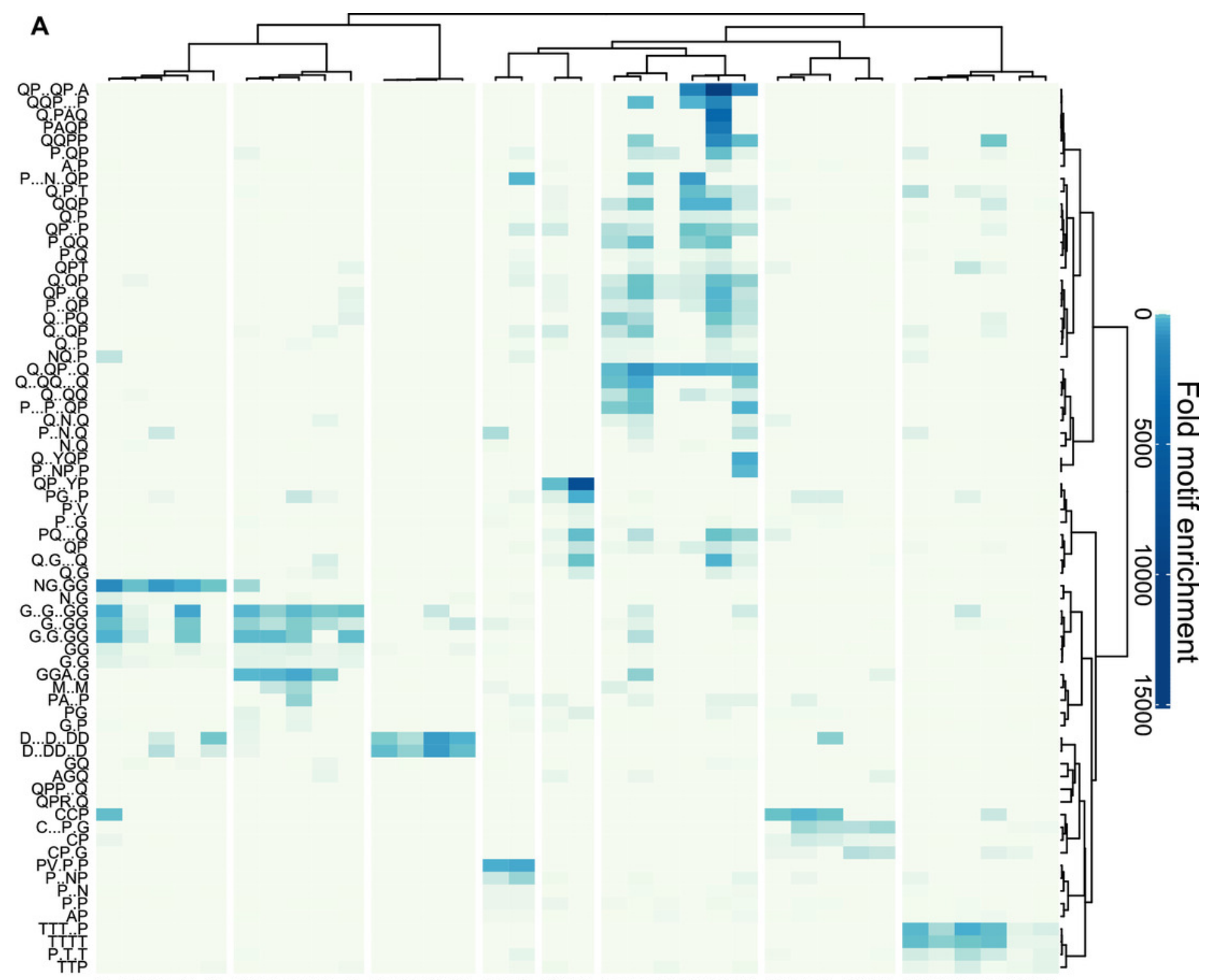

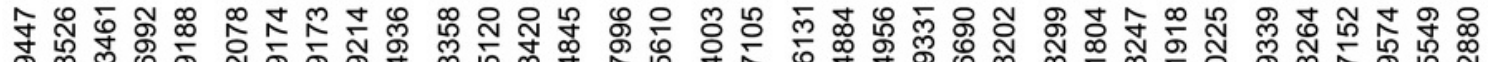

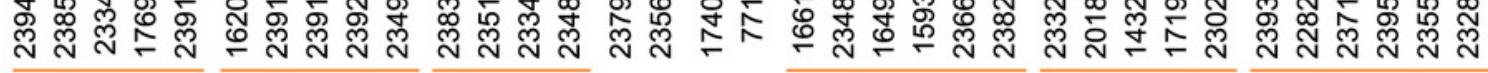 Cluster 1 Cluster 2 Cluster 3

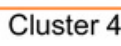

Cluster 4
Cluster 5 Cluster 6

B

Cluster 1

R.NG.GG G..NGGA.G

S.GG.G.G S.G.G.G.G..S

GG.GG.GN.GMM

NG.GG

C

SG G.GNG.N

G.YGG.G..GG.E

AG..GG.GGM

Couser 3
D...DD
D.NDD
B.DD..D

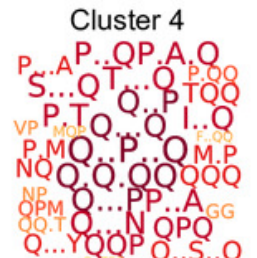

Cluster 5
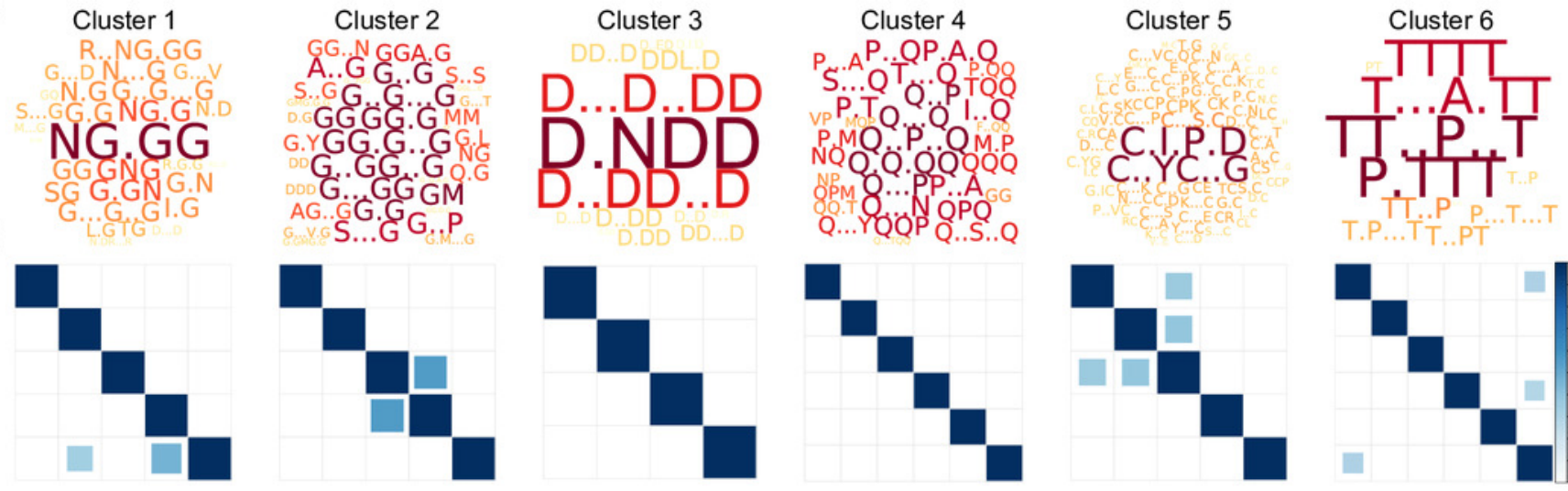

C.YP.D
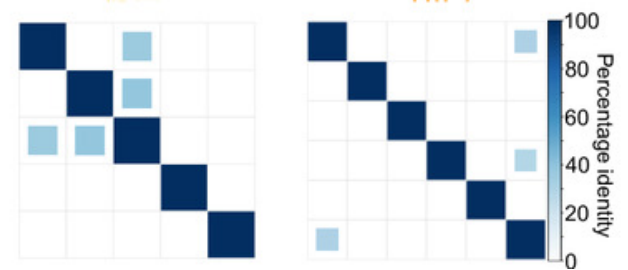